\title{
OPTIMAL MULTICOMMODITY FLOW THROUGH THE COMPLETE GRAPH WITH RANDOM EDGE CAPACITIES
}

\author{
MUSTAFA KHANDWAWALA*** AND \\ RAJESH SUNDARESAN, ${ }^{* * * *}$ Indian Institute of Science
}

\begin{abstract}
We consider a multicommodity flow problem on a complete graph whose edges have random, independent, and identically distributed capacities. We show that, as the number of nodes tends to infinity, the maximum utility, given by the average of a concave function of each commodity flow, has an almost-sure limit. Furthermore, the asymptotically optimal flow uses only direct and two-hop paths, and can be obtained in a distributed manner.
\end{abstract}

Keywords: Complete graph; fairness; multicommodity flow; routeing; utility

2000 Mathematics Subject Classification: Primary 05C21

Secondary $05 \mathrm{C} 80 ; 90 \mathrm{C} 25$

\section{Introduction}

Flow maximisation on a graph is a central problem in graph theory and optimisation. The single-source, single-sink flow problem has been studied extensively and several algorithms have been developed for obtaining the maximum flow. An important flow problem that can be used to model realistic networks is the multicommodity flow, in which there is simultaneous flow between each source-destination pair. In this paper we consider an edge-capacitated undirected graph. We associate a utility to the flow between each source-destination vertex pair, and seek to optimise the average utility of the flows. We first describe the problem and its solution. Towards the end of this section we indicate how our problem arises in practice.

For a given source $v$ and destination $w$, the associated flow between them is conserved at all vertices except $v$ and $w$. Writing $\varphi_{v w}(e)$ as the absolute value of this flow on an edge $e$, the volume of this $v w$ flow is given by

$$
f_{v w}=\sum_{\{e: e \text { incident on } v\}} \varphi_{v w}(e)=\sum_{\{e: e \text { incident on } w\}} \varphi_{v w}(e) .
$$

Assume that each pair of vertices of the graph forms a source-destination pair with the sourcedestination labelling chosen arbitrarily. Then, given capacities $C(e)$ for edges $e$, we say that

\footnotetext{
Received 5 October 2009.

* Postal address: Department of Electrical Communication Engineering, Indian Institute of Science, Bangalore 560012, India.

This work was supported by the Department of Science and Technology under grant DSTO748, and by the University Grants Commission under grant Part (2B) UGC-CAS-(Ph.IV).

** Email address: mustafa@ece.iisc.ernet.in

*** Email address: rajeshs@ece.iisc.ernet.in
} 
the flow profile $\left\{f_{v w}\right\}_{v, w}$ obtained via $\left\{\varphi_{v w}(e)\right\}_{e, v, w}$ is feasible if

$$
\sum_{\{v, w\}} \varphi_{v w}(e) \leq C(e) \text { for all } e .
$$

We consider the complete $n$-vertex graph $G_{n}$ with random edge capacities, and quantify the behaviour of the average utility as $n \rightarrow \infty$. Such a model was studied by Aldous et al. [1] under the setting of a uniform multicommodity flow, where all flows are required to be of the same volume. We interchangeably use the notation $C(e)$ or $C_{v w}$ for the capacity of an edge $e$ incident on vertices $v$ and $w$. We assume that the capacities $C_{v w}$ are independent and identically distributed (i.i.d.) copies of a reference random variable $C$ that takes values in a set $\mathcal{C} \subseteq \mathbb{R}^{+}$ and satisfies $0<\mathrm{E}[C]<\infty$.

For a given feasible flow profile $\left\{f_{v w}\right\}_{v, w}$, we define the utility of the flow profile to be

$$
U_{n}=\frac{1}{a_{n}} \sum_{\{v, w\}} \zeta\left(f_{v w}\right),
$$

where $\zeta: \mathbb{R}^{+} \rightarrow \mathbb{R} \cup\{-\infty\}$ is a strictly concave, increasing utility function with a continuous first derivative, $\zeta(x)>-\infty$ if $x>0$, and $a_{n}=\left(\begin{array}{l}n \\ 2\end{array}\right)$ is the number of edges. The maximum utility is denoted by

$$
\rho_{n}=\sup \left\{U_{n} \mid\left\{f_{v w}\right\}_{v, w} \text { feasible }\right\} .
$$

Examples of such utility functions are the so-called $\alpha$-fair utility functions [10]

$$
\zeta_{\alpha}(x)= \begin{cases}\frac{x^{1-\alpha}}{1-\alpha}, & \alpha \in[0, \infty), \alpha \neq 1, \\ \log x, & \alpha=1 .\end{cases}
$$

As $\alpha \rightarrow \infty$, we have

$$
\lim _{\alpha \rightarrow \infty} U_{n}^{1 /(1-\alpha)}=\min _{\{v, w\}} f_{v w},
$$

and the problem reduces to the uniform multicommodity flow of Aldous et al. [1]. The solution to this problem may be thought of as a max-min fair solution. Aldous et al. [1] showed that

- $\rho_{n}$ for the uniform flow case converges in probability to a constant that depends on the distribution of $C$;

- each flow may be routed through only direct and two-hop paths.

Their proof technique does not appear to be amenable to a distributed implementation.

Instead of choosing arbitrary source-destination labellings for a given pair of vertices as in our model, Aldous et al. [1] considered every ordered pair as a source-destination pair. We can frame our problem in that context by interpreting $f_{v w}$ as the volume of flow from $v$ to $w$ and $f_{w v}$ as the volume of flow from $w$ to $v$, and using ordered pairs $(v, w)$ in the definition of $U_{n}$. However, concavity of $\zeta$ implies that flows in either direction should be equal for optimality. We therefore do not distinguish between $f_{v w}$ and $f_{w v}$, and we let $f_{v w}$ denote the net flow between $v$ and $w$ with one of them arbitrarily taken as the source and the other as the destination. 
Our main results are the following.

- $\rho_{n}$ converges with probability 1 to a constant $\mathrm{E}[\zeta(h(C))]$, where $h$ is the piecewise linear function truncated at $a$ and saturated at $b$ :

$$
h(x)= \begin{cases}a, & x \leq a \\ x, & a \leq x \leq b, \\ b, & x \geq b .\end{cases}
$$

The constants $a$ and $b$ depend on the distribution of $C$.

- Each flow $f_{v w}$ in the asymptotically optimal flow profile is given by $h\left(C_{v w}\right)$, a function of the capacity of the direct edge alone.

- Each flow requires only direct paths and two-hop paths.

- Our solution to find the flow profile is amenable to a distributed implementation.

Multicommodity flow problems were introduced in [9], and an algorithm for obtaining maxmin fair optimal flow was described. Such problems arise in computer communication and wireless networks. Algorithms for solving multicommodity flow problems with fixed demands and capacities were described in [6] and [8]. Flows over networks with random edge capacities, where the capacities form a stochastic process with time as a parameter, were studied in the monograph [3] and the references therein. One objective that was considered was to maximise the sum of concave utilities [10] arising from flow values. See [7] for a nonrandom version where there is only one route per flow. Georgiadis et al. [3] considered several generalisations with multiple routes, dynamic routeing, random arrivals, and queues. The problem considered by Aldous et al. [1] and ours in this paper may be regarded as an asymptotic version of the simplest of these problems, with no queues and no time variations, but with the network size growing to infinity and one commodity per pair of vertices. This tractable asymptotic version may provide useful bounds for other intractable problems. Related problems along these asymptotic lines are those of flows between the top and bottom surfaces of a lattice with random edge capacities [2], [4], [11].

The rest of the paper is organised as follows. In Section 2 we solve the problem when $\zeta$ is linear. In Section 3 we provide conditions that ensure achievability of a utility when $\zeta$ is strictly concave, and we describe a distributed method to obtain the corresponding feasible flow. We optimise the lower bound subject to these conditions in Section 4, and prove that this is, in fact, optimal in Section 5. Some final remarks in Section 6 conclude the paper.

\section{Linear utility}

In this section we consider the linear utility function $\zeta(x)=x$. Note that this is not strictly concave. However, it turns out that the optimal flow profile for this problem is also optimal for some strictly concave $\zeta \mathrm{s}$, as will be highlighted later.

Theorem 1. If $\zeta(x) \equiv x$ then $\rho_{n} \rightarrow \mathrm{E}[C]$ as $n \rightarrow \infty$ with probability 1 .

Proof. Let $f_{v w}=C_{v w}$ for all $\{v, w\}$. This flow profile is clearly feasible because each flow uses only the direct link to its capacity. For this allocation,

$$
U_{n}=\frac{1}{a_{n}} \sum_{\{v, w\}} C_{v w} .
$$


Next, let $\left\{\varphi_{v w}(e)\right\}_{e, v, w}$ form a feasible flow. The capacity constraints are

$$
\sum_{\{v, w\}} \varphi_{v w}(e) \leq C(e) \text { for all } e .
$$

Summing over all edges $e$ and interchanging the summations, we obtain

$$
\sum_{\{v, w\}} \sum_{e} \varphi_{v w}(e) \leq \sum_{e} C(e)
$$

We also have

$$
f_{v w}=\sum_{\{e: e \text { incident on } v\}} \varphi_{v w}(e) \leq \sum_{e} \varphi_{v w}(e) \text { for all }\{v, w\} .
$$

Summing over all pairs $\{v, w\}$ and using (2), we obtain, after dividing by $a_{n}$,

$$
U_{n}=\frac{1}{a_{n}} \sum_{\{v, w\}} f_{v w} \leq \frac{1}{a_{n}} \sum_{e} C(e)
$$

for any feasible flow. From (1), the upper bound in (3) is achievable, and, hence,

$$
\rho_{n}=\frac{1}{a_{n}} \sum_{e} C(e)
$$

which converges to $\mathrm{E}[C]$ as $n \rightarrow \infty$ with probability 1 .

\section{Achievability of the flow}

When $\zeta$ is linear, we showed in Section 2 that the optimal flow is achieved by using only the direct link for each flow at its capacity. While this yields an efficient solution, the flow profile can be unfair. On the other hand, as proved in [1], the maximally fair asymptotically optimal flow profile is obtained using only direct and two-hop links. As such, it seems natural that the optimal flow in the case of a concave utility function, which enables operations between the two extremes, need not use more than two hops.

Now suppose that the flow volume $f_{v w}$ depends only on the capacity of the direct link $C_{v w}$ for all pairs $\{v, w\}$, i.e. $f_{v w}=h\left(C_{v w}\right)$ for some $h: \mathcal{C} \rightarrow \mathbb{R}^{+}$. In this section we obtain a sufficient condition (see (13), below) for such a flow to be feasible. Asymptotic optimality of such a flow is established in Section 5.

For the uniform flow case, we set $h\left(C_{v w}\right)=\phi$ for all $\{v, w\}$ and remark that condition (13), below, reduces to the necessary and sufficient condition for the feasible uniform flow, as proved in [1]. Thus, the uniform multicommodity flow arises as a special case and the proof here serves as an alternative to the proof of achievability given by Aldous et al. [1]. Our proof is elementary and is amenable to a distributed implementation.

\subsection{Feasibility of certain integer flows}

Here we show the achievability of certain integer flows with integer capacity constraints. This serves as the primary tool used to prove the main result of this paper. The proof is a modification of a procedure of Aldous et al. [1]. 
Lemma 1. Let $C$ and $F$ be random variables taking only nonnegative integer values. Let $M<\infty$ be an upper bound for both $C$ and $F$. Let $\left\{\left(C_{v w}, F_{v w}\right)\right\}_{v, w}$ be a set of i.i.d. pairs of random variables with each pair having the distribution of $(C, F)$. If

$$
\mathrm{E}\left[(C-F)^{+}\right]-2 \mathrm{E}\left[(F-C)^{+}\right]>0,
$$

the flow on $G_{n}$ obtained by setting $f_{v w}=F_{v w}$ for all $\{v, w\}$ is feasible for all but finitely many $n$, with probability 1 .

Proof. Let $C$ and $F$ be such that (4) holds.

If $C_{v w} \geq F_{v w}$ for a given pair $\{v, w\}$, we use only the direct edge $v w$ for the flow $f_{v w}$. Then, $C_{v w}-F_{v w}$ is the remaining capacity along edge $v w$. If $F_{v w}>C_{v w}$, we use the entire capacity $C_{v w}$ of the direct edge for a part of $f_{v w}$. Then, $F_{v w}-C_{v w}$ is the remaining flow demand between $v$ and $w$.

We decompose the original problem into $M$ separate flow problems by constructing $M$ graphs $P_{1}, P_{2}, \ldots, P_{M}$, each with $n$ vertices, as follows. For each vertex pair $\{v, w\}$, such that $F_{v w}>C_{v w}$, choose an $F_{v w}-C_{v w}$ size subset $S_{1}$ of $\{1,2, \ldots, M\}$ uniformly and independently of other vertex pairs. For each $i \in S_{1}$, put a scarlet edge between $v$ and $w$ in graph $P_{i}$. Similarly, for each pair $\{v, w\}$, such that $C_{v w} \geq F_{v w}$, choose a $C_{v w}-F_{v w}$ size subset $S_{2}$ of $\{1,2, \ldots, M\}$ uniformly and independently of other vertex pairs. For each $i \in S_{2}$, put a blue edge between $v$ and $w$ in graph $P_{i}$.

Now focus on one particular graph $P_{i}$. For a fixed vertex pair $\{v, w\}$, there is a scarlet edge between $v$ and $w$ in $P_{i}$ with probability $p_{s}$ given in (5), below, a blue edge with probability $p_{b}$ given in (6), below, and no edge with the remaining probability $1-p_{s}-p_{b}$. Also, this happens independently for all vertex pairs. As $\left(\begin{array}{c}M-1 \\ j-1\end{array}\right) /\left(\begin{array}{c}M \\ j\end{array}\right)=j / M$ is the probability that a particular $i \in S_{1}$ given $\left|S_{1}\right|=j$, and analogously for $i \in S_{2}$ given $\left|S_{2}\right|=j$, we have

$$
\begin{aligned}
& p_{s}=\sum_{j=1}^{M} \operatorname{Pr}\left\{F_{v w}-C_{v w}=j\right\} \frac{j}{M}=\frac{\mathrm{E}\left[(F-C)^{+}\right]}{M}, \\
& p_{b}=\sum_{j=0}^{M} \operatorname{Pr}\left\{C_{v w}-F_{v w}=j\right\} \frac{j}{M}=\frac{\mathrm{E}\left[(C-F)^{+}\right]}{M} .
\end{aligned}
$$

By the assumption in (4) we have $p_{b}>2 p_{s}$.

In the graph $P_{i}$, a scarlet edge between vertices $v$ and $w$ indicates a yet to be fulfilled unit demand for the $v w$ flow, and a blue edge between $v$ and $w$ indicates the availability of unit capacity along the edge $v w$. Thus, if in all the $P_{i}, i=1,2, \ldots, M$, the demands along scarlet edges can be satisfied via the available capacities along blue edges, the flow $\left\{f_{v w}=F_{v w}\right\}_{v, w}$ can be achieved.

Such a problem is solved in [1] using a packing result to form edge-disjoint triangles, each containing one scarlet and two blue edges, which cover all scarlet edges. Here we use an alternate method. The argument proceeds roughly as follows. A blue edge $v w$ can potentially serve a $v z$ flow for a vertex $z$ if $v z$ is a scarlet edge and $w z$ is a blue edge. Similar is the case when $w z$ is scarlet but $v z$ is blue. By the nature of the colouring, the number of such vertices is a random variable having binomial distribution with parameters $\left(n-2,2 p_{s} p_{b}\right)$. The flow between two vertices having a scarlet edge between them can be served via a vertex connected to both by blue edges. The number of such vertices is a random variable having binomial distribution with parameters $\left(n-2, p_{b}^{2}\right)$. Dividing the flow across all such two-hop routes and 
using the concentration of the binomial distribution, we can get the required flows with high probability if $p_{b}^{2}>2 p_{s} p_{b}$.

Formally, let $v w$ be a blue edge. Define $N_{v w}$ as the number of vertices $t \neq v, w$ such that $t$ is connected to $v$ and $w$ by one scarlet and one blue edge.

Now, consider a scarlet edge $v z$. For all vertices $w \neq v, z$ connected to $v$ and $z$ by two blue edges, allocate a fractional flow of $1 / \max \left(N_{v w}, N_{z w}\right)$ through the two-hop path $v-w-z$. Do this for all scarlet edges. Then the flow through any blue edge is not greater than 1 . The flow allocated for the scarlet $v z$ is given by the random variable

$$
\begin{aligned}
R_{v z} & =\sum_{w \neq v, z} \mathbf{1}_{\{v w=\text { blue }\}} \mathbf{1}_{\{z w=\text { blue }\}} \frac{1}{\max \left(N_{v w}, N_{z w}\right)} \\
& \geq J_{v z} \frac{1}{\max _{w \neq v, z}\left\{\max \left(N_{v w}, N_{z w}\right)\right\}}
\end{aligned}
$$

where $J_{v z}=\sum_{w \neq v, z} \mathbf{1}_{\{v w=\text { blue }\}} \mathbf{1}_{\{z w=\text { blue }\}}$ is a binomial random variable with parameters $\left(n-2, p_{b}^{2}\right)$. Note that, for a fixed scarlet $v z$ and fixed $w$ with blue $v w$ and $z w, N_{v w}-1$ is a binomial $\left(n-3,2 p_{s} p_{b}\right)$ random variable, conditioned on $z$ contributing 1 to the $N_{v w}$ count. Then,

Since $p_{b}>2 p_{s}$, we have $p_{b}^{2}-2 p_{s} p_{b}>0$. Choose $\varepsilon$ such that $0<\varepsilon \leq\left(p_{b}^{2}-2 p_{s} p_{b}\right) / 2$.

$$
\frac{p_{b}^{2}-\varepsilon}{2 p_{s} p_{b}+\varepsilon} \geq 1
$$

From (7) and (8), the event

$$
\left\{R_{v z}<1\right\} \Longrightarrow\left\{J_{v z} \leq(n-2)\left(p_{b}^{2}-\varepsilon\right)\right\} \cup\left\{\max _{w \neq v, z}\left\{\max \left(N_{v w}, N_{z w}\right)\right\} \geq(n-2)\left(2 p_{s} p_{b}+\varepsilon\right)\right\} .
$$

By Bernstein's inequality [5, p. 31],

$$
\operatorname{Pr}\left\{J_{v z} \leq(n-2)\left(p_{b}^{2}-\varepsilon\right)\right\} \leq \mathrm{e}^{-(n-2) \varepsilon^{2} / 4} .
$$

Noting that

$$
(n-2)\left(2 p_{s} p_{b}+\varepsilon\right)-1 \geq(n-3)\left(2 p_{s} p_{b}+\frac{1}{2} \varepsilon\right)
$$

for all $n>3+2 / \varepsilon$, we obtain

$$
\operatorname{Pr}\left\{\max _{w \neq v, z}\left\{\max \left(N_{v w}, N_{z w}\right)\right\} \geq(n-2)\left(2 p_{s} p_{b}+\varepsilon\right)\right\} \leq 2(n-2) \mathrm{e}^{-(n-3) \varepsilon^{2} / 16}
$$

by the application of Bernstein's inequality and the union bound. Using (10) and (11) in (9), we obtain

$$
\operatorname{Pr}\left\{R_{v z}<1\right\} \leq \mathrm{e}^{-(n-2) \varepsilon^{2} / 4}+2(n-2) \mathrm{e}^{-(n-3) \varepsilon^{2} / 16} \leq 2 n \mathrm{e}^{-(n-3) \varepsilon^{2} / 16} .
$$

Since there are a maximum $n(n-1) / 2 \leq n^{2} / 2$ scarlet edges, we have

$$
\operatorname{Pr}\left\{R_{v z}<1 \text { for some scarlet edge } v z\right\} \leq \frac{n^{2}}{2} 2 n \mathrm{e}^{-(n-3) \varepsilon^{2} / 16} .
$$


Using the same procedure over all $M$ graphs, and denoting by $A_{n}$ the event that the flow profile $\left\{f_{v w}=F_{v w}\right\}_{v, w}$ on $G_{n}$ is not feasible, i.e. there is some scarlet edge $v z$ in one of the $M$ graphs with $R_{v z}<1$, we obtain

$$
\operatorname{Pr}\left\{A_{n}\right\} \leq M n^{3} \mathrm{e}^{-(n-3) \varepsilon^{2} / 16} .
$$

From (12), $\sum_{n=1}^{\infty} \operatorname{Pr}\left\{A_{n}\right\}<\infty$. This ensures, by the Borel-Cantelli lemma [5, p. 288], that the probability of $A_{n}$ occurring infinitely often is 0 . Hence, the flow $\left\{f_{v w}=F_{v w}\right\}_{v, w}$ on $G_{n}$ is feasible for all but finitely many $n$, with probability 1 .

\subsection{Sufficient condition for a feasible flow}

Lemma 2. Let $h: \mathcal{C} \rightarrow \mathbb{R}^{+}$be a function such that $\inf _{x \in \mathcal{C}} h(x)>0$ and $\sup _{x \in \mathcal{C}} h(x)<\infty$. If

$$
\mathrm{E}\left[(C-h(C))^{+}\right]-2 \mathrm{E}\left[(h(C)-C)^{+}\right] \geq 0
$$

then $\lim \inf _{n \rightarrow \infty} \rho_{n} \geq \mathrm{E}[\zeta(h(C))]$ with probability 1 .

Proof. First observe that the expectation in (13) exists and is finite because $\mathrm{E}|h(C)-C| \leq$ $\mathrm{E} h(C)+\mathrm{E} C$, both of which exist and are finite. The expectation $\mathrm{E}[\zeta(h(C))]$ exists by Jensen's inequality and the assumption on $\zeta$ that $\zeta(x)>-\infty$ if $x>0$.

Choose $\delta$ such that $0<2 \delta<\inf _{x \in \mathcal{C}} h(x)$, and choose an integer $k$ large enough that $k>2 / \delta$ and $k>\sup _{x \in \mathcal{C}} h(x)$. Define the random variables

$$
C^{(k)}=\frac{1}{k}\left\lfloor\min \left(k C, k^{2}\right)\right\rfloor
$$

and

$$
F^{(k)}=\frac{1}{k}\lfloor k h(C)-k \delta-1\rfloor .
$$

Observe that

$$
0 \leq C^{(k)} \leq C
$$

and

$$
0 \leq F^{(k)} \leq \frac{1}{k}(k h(C)-k \delta-1) \leq k-\delta .
$$

Moreover, $\left(k C_{v w}^{(k)}, k F_{v w}^{(k)}\right)$ are i.i.d. nonnegative integer quantities, so we are in a position to apply Lemma 1 if we can verify (4) for $\left(C^{(k)}, F^{(k)}\right)$. To do this, we may write the expectation in (4) as an integral over $\{C \leq k\}$ and $\{C>k\}$, and use (14) and (16) to obtain

$$
\begin{aligned}
& \mathrm{E}\left[\left(C^{(k)}-F^{(k)}\right)^{+}-2\left(F^{(k)}-C^{(k)}\right)^{+}\right] \\
& \geq \frac{1}{k} \int_{c \leq k}\left[((k c-1)-(k h(c)-k \delta-1))^{+}-2((k h(c)-k \delta-1)-(k c-1))^{+}\right] \mathrm{d} \mu(c) \\
&+\int_{c>k}\left[(k-k+\delta)^{+}-2(k-\delta-k)^{+}\right] \mathrm{d} \mu(c) \\
&= \int_{c \leq k}\left[(c-h(c)+\delta)^{+}-2(h(c)-\delta-c)^{+}\right] \mathrm{d} \mu(c)+\int_{c>k} \delta \mathrm{d} \mu(c) \\
& \geq \int_{c \leq k}\left[(c-h(c))^{+}-2(h(c)-c)^{+}+\delta\right] \mathrm{d} \mu(c)+\int_{c>k} \delta \mathrm{d} \mu(c) \\
&= \int_{c \leq k}\left[(c-h(c))^{+}-2(h(c)-c)^{+}\right] \mathrm{d} \mu(c)+\delta,
\end{aligned}
$$


where the second inequality follows because

$$
(x+\delta)^{+}-2(x+\delta)^{-} \geq x^{+}-2 x^{-}+\delta \quad \text { for all } x \in \mathbb{R} .
$$

By the dominated convergence theorem,

$$
\lim _{k \rightarrow \infty} \int_{c \leq k}\left[(c-h(c))^{+}-2(h(c)-c)^{+}\right] \mathrm{d} \mu(c)=\mathrm{E}\left[(C-h(C))^{+}-2(h(C)-C)^{+}\right] \geq 0 .
$$

So choose $k$ large enough that

$$
\int_{c \leq k}\left[(c-h(c))^{+}-2(h(c)-c)^{+}\right] \mathrm{d} \mu(c)>-\delta .
$$

Its substitution into (17) implies that

$$
\mathrm{E}\left[\left(C^{(k)}-F^{(k)}\right)^{+}-2\left(F^{(k)}-C^{(k)}\right)^{+}\right]>0 .
$$

Hence, by Lemma 1 , the flow $\left\{f_{v w}=k F_{v w}^{(k)}\right\}_{v, w}$ is feasible for all but finitely many $n$, with probability 1 when we have integer capacities $\left\{k C_{v w}^{(k)}\right\}_{v, w}$. Scaling by $1 / k$ and noting that $C^{(k)} \leq C$, the flow $\left\{f_{v w}=F_{v w}^{(k)}\right\}_{v, w}$ is feasible for all large enough $k$ and all but finitely many $n$, with probability 1 . For this flow profile, the utility is

$$
U_{n}^{(k)}=\frac{1}{a_{n}} \sum_{\{v, w\}} \zeta\left(F_{v w}^{(k)}\right) .
$$

Since $F^{(k)} \geq h(C)-2 \delta>0$, as is easily verified, and $\zeta$ is an increasing function,

$$
\begin{aligned}
U_{n}^{(k)} & \geq \frac{1}{a_{n}} \sum_{\{v, w\}} \zeta\left(h\left(C_{v w}\right)-2 \delta\right) \\
& \geq \frac{1}{a_{n}} \sum_{\{v, w\}}\left[\zeta\left(h\left(C_{v w}\right)\right)-2 \delta \zeta^{\prime}\left(h\left(C_{v w}\right)-2 \delta\right)\right] \\
& \geq \frac{1}{a_{n}} \sum_{\{v, w\}}\left[\zeta\left(h\left(C_{v w}\right)\right)-2 \delta \zeta^{\prime}\left(\inf _{x \in \mathcal{C}} h(x)-2 \delta\right)\right] .
\end{aligned}
$$

The second inequality above follows from the strict concavity of $\zeta$. Since

$$
\delta \zeta^{\prime}\left(\inf _{x \in \mathcal{C}} h(x)-2 \delta\right) \rightarrow 0
$$

as $\delta \rightarrow 0$, we have

$$
U_{n}^{(k)} \geq \frac{1}{a_{n}} \sum_{\{v, w\}} \zeta\left(h\left(C_{v w}\right)\right)-\varepsilon
$$

for any $\varepsilon>0$. Noting that $\rho_{n} \geq U_{n}^{(k)}$, the event

$$
B_{\varepsilon}:=\left\{\liminf _{n \rightarrow \infty} \rho_{n} \geq \mathrm{E}[\zeta(h(C))]-\varepsilon\right\}
$$

occurs with probability 1 . Consequently, the event

$$
B=\bigcap_{m=1}^{\infty} B_{1 / m}=\left\{\liminf _{n \rightarrow \infty} \rho_{n} \geq \mathrm{E}[\zeta(h(C))]\right\}
$$

also occurs with probability 1 . 


\subsection{A distributed implementation}

The proofs of Lemma 1 and Lemma 2 provide a randomised algorithm to obtain the feasible flow, which can be implemented in a distributed manner. The first step is to obtain an integer approximation as defined in (14) and (15). We may need to choose $k$ large enough to get a utility sufficiently close to $\mathrm{E}[\zeta(h(C))]$. Then, we use the algorithm in the proof of Lemma 1 to obtain a routeing for this flow. Note that randomisation arises from the choice of the subsets that determine the edge colours in the $M$ subgraphs. Here, we highlight the distributed nature of this algorithm.

The information available at an edge is assumed to be available also at its endvertices. These include the capacity $C^{(k)}$, the flow requirement $F^{(k)}$, the presence or absence of the edge in each of the $M$ graphs, and their colours. Fix one of the $M$ graphs. Then each vertex will query each of its neighbours to obtain $N_{v w}$ for each vertex $w$ such that $v w$ is blue. Then $v$ exchanges the information on edge colours with each vertex $z$ with $v z$ scarlet to determine the $v z$ flow on the path $v-w-z$ where $v w$ and $w z$ are both blue. This happens for each of the $M$ graphs. Even though every node exchanges data with every other node, the graph is complete, the flow values and routes are determined based on locally available information.

\section{Optimisation of the lower bound}

Having found a sufficient condition (13) for feasible flow in Lemma 2, we optimise the utility over all such functions $h$. Recall that $\zeta$ is a strictly concave function. Consider the following functional optimisation problem:

$$
\max _{\left\{h: \mathcal{C} \rightarrow \mathbb{R}^{+}\right\}} \mathrm{E}[\zeta(h(C))]
$$

subject to $\mathrm{E}\left[(C-h(C))^{+}\right]-2 \mathrm{E}\left[(h(C)-C)^{+}\right] \geq 0$.

Let $h^{*}$ be the optimising function. We will show that, under the stated assumptions on $\zeta, h^{*}$ exists, so that the use of max in (18) is justified.

Let $\bar{\theta}=\lim _{x \downarrow 0} \zeta^{\prime}(x)$ and $\underline{\theta}=\lim _{x \uparrow \infty} \zeta^{\prime}(x)$. We have $0 \leq \underline{\theta} \leq \bar{\theta}$, and we may assume that $\underline{\theta}<\infty$ and $\bar{\theta}>0$. Define

$$
\psi(h)=\mathrm{E}[\zeta(h(C))]
$$

and

$$
\xi(h)=\mathrm{E}\left[(C-h(C))^{+}\right]-2 \mathrm{E}\left[(h(C)-C)^{+}\right] .
$$

Proposition 1. If $\bar{\theta} \leq 2 \underline{\theta}$ then $h^{*}(c)=c$ for all $c \in \mathcal{C}$.

Proof. Choose $\lambda>0$ such that

$$
\frac{\bar{\theta}}{2} \leq \lambda \leq \underline{\theta} .
$$

Consider the function

$$
\begin{aligned}
w(h, \lambda) & =\psi(h)+\lambda \xi(h) \\
& =\mathrm{E}\left[\zeta(h(C))+\lambda(C-h(C))^{+}-2 \lambda(h(C)-C)^{+}\right] .
\end{aligned}
$$

We first maximise $w(h, \lambda)$ over all functions $h: \mathcal{C} \rightarrow \mathbb{R}^{+}$for a fixed $\lambda$. Let the optimising function exist and be given by $h_{\lambda}$, and suppose that $\lambda$ is such that $\xi\left(h_{\lambda}\right)=0$. Then, since $w\left(h_{\lambda}, \lambda\right) \geq w(h, \lambda)$, we have $\psi\left(h_{\lambda}\right)+\lambda \xi\left(h_{\lambda}\right) \geq \psi(h)+\lambda \xi(h)$. Thus, $\psi\left(h_{\lambda}\right) \geq \psi(h)+$ $\lambda \xi(h) \geq \psi(h)$ over all functions $h$ that satisfy $\xi(h) \geq 0$. Thus, $h_{\lambda}$ is the optimising $h^{*}$ for 
problem (18). We now prove the existence of such a $\lambda$ and $h_{\lambda}$. We may write (21) as

$$
w(h, \lambda)=\int_{\mathcal{C}}\left[\zeta(h(c))+\lambda(c-h(c))^{+}-2 \lambda(h(c)-c)^{+}\right] \mathrm{d} \mu(c) .
$$

Maximising $w(h, \lambda)$ is equivalent to maximising the integrand $\zeta(h(c))+\lambda(c-h(c))^{+}-$ $2 \lambda(h(c)-c)^{+}$pointwise for each $c \in[0, \infty)$. Thus, writing $h(c)=f$, we look to maximise

$$
\zeta(f)+\lambda(c-f)^{+}-2 \lambda(f-c)^{+}
$$

over $f \geq 0$ for a fixed $c$.

Define

$$
g_{1}(f)=\zeta(f)-2 \lambda(f-c)
$$

and

$$
g_{2}(f)=\zeta(f)+\lambda(c-f) .
$$

The maximum value of (22) can be written in terms of $g_{1}$ and $g_{2}$ as

$$
\max \left\{\sup _{0 \leq f<c} g_{2}(f), \sup _{f>c} g_{1}(f), \zeta(c)\right\} .
$$

The functions $g_{1}(f)$ and $g_{2}(f)$ are strictly concave functions in $f$. By the conditions on the slopes in the hypothesis and by (20), we have $g_{1}^{\prime}(f) \leq 0$ and $g_{2}^{\prime}(f) \geq 0$; so $g_{1}(f)$ is maximised at $f=0$ and $g_{2}(f)$ is maximised at $f=\infty$. Because of the concavity of $g_{1}(f)$ and $g_{2}(f)$, we have

$$
\begin{gathered}
g_{1}(0) \geq g_{1}(c)=\zeta(c) \geq g_{1}(f) \quad \text { for } c \leq f, \\
g_{2}(\infty) \geq g_{2}(c)=\zeta(c) \geq g_{2}(f) \quad \text { for } 0 \leq f \leq c .
\end{gathered}
$$

The above equations imply that $\sup _{0 \leq f<c} g_{2}(f) \leq \zeta(c)$ and $\sup _{f>c} g_{1}(f) \leq \zeta(c)$. Thus, for each fixed $c$, the optimal value of $(22)$ is $\zeta(c)$ and it is achieved by setting $f=c$. Hence, the optimisation function $h_{\lambda}(c)=c$ for all $c \in \mathcal{C}$ and any $\lambda$ that satisfies (20). Furthermore, $\xi\left(h_{\lambda}\right)=0$, and, hence, $h^{*}(c) \equiv c$ is the optimising function.

For the other case, $\bar{\theta}>2 \underline{\theta}$, we need the following definition. Define

$$
\operatorname{SAT}(c, a, b)=\min (\max (a, c), b)
$$

for given $a \leq b$ and

$$
p_{\lambda}=\operatorname{SAT}\left(c, \zeta^{\prime-1}(2 \lambda), \zeta^{\prime-1}(\lambda)\right)
$$

for $\lambda \in[\underline{\theta}, \bar{\theta} / 2]$.

Proposition 2. If $\bar{\theta}>2 \underline{\theta}$ then

$$
h^{*}(c)=p_{\lambda^{*}},
$$

where $\lambda^{*} \in[\underline{\theta}, \bar{\theta} / 2] \cap[0, \infty)$ is such that $\xi\left(p_{\lambda^{*}}\right)=0$.

Proof. Choose $\lambda>0$ such that $\underline{\theta} \leq \lambda \leq \bar{\theta} / 2$. We proceed as in the proof of Proposition 1 to maximise (22) for a fixed $c \in[0, \infty)$.

In this case, $g_{1}(f)$ and $g_{2}(f)$, defined in (23) and (24), have unique maxima $g_{1}\left(f_{1}\right)$ and $g_{2}\left(f_{2}\right)$ obtained at $f_{1}=\zeta^{\prime-1}(2 \lambda)$ and $f_{2}=\zeta^{\prime-1}(\lambda)$, respectively. Observe that $f_{1}<f_{2}$. 
Because of the concavity of $g_{1}(f)$ and $g_{2}(f)$, we have the following inequalities under the specified cases on $c$ :

$$
\begin{array}{ll}
g_{1}\left(f_{1}\right) \geq g_{1}(c)=\zeta(c) \geq g_{1}(f) & \text { for } f_{1} \leq c \leq f, \\
g_{2}\left(f_{2}\right) \geq g_{2}(c)=\zeta(c) \geq g_{2}(f) & \text { for } f \leq c \leq f_{2} .
\end{array}
$$

For $f \leq c \leq f_{1}$, and since $f_{1} \leq f_{2}$, we have, from (27), the condition

$$
g_{1}\left(f_{1}\right) \geq \zeta(c) \geq g_{2}(f) \text { for } f \leq c \leq f_{1} .
$$

Analogously, for $f_{2} \leq c \leq f$, and since $f_{1} \leq f_{2}$, we have, from (26), the condition

$$
g_{2}\left(f_{2}\right) \geq \zeta(c) \geq g_{1}(f) \text { for } f_{2} \leq c \leq f .
$$

For $c<f_{1}, f^{*}=f_{1}$ maximises (25) because of (28). Similarly, for $c>f_{2}, f^{*}=f_{2}$ maximises (25) because of (29). For $f_{1} \leq c \leq f_{2}, f^{*}=c$ maximises (25) because of (26) and (27). Hence, $h_{\lambda}=p_{\lambda}$ maximises $w(h, \lambda)$.

We next check that there exists a $\lambda^{*} \in[\underline{\theta}, \bar{\theta} / 2]$ with $\xi\left(h_{\lambda^{*}}\right)=0$. Note that

$$
\xi\left(h_{\lambda}\right)=\int_{\mathcal{C}}\left[\left(c-\zeta^{\prime-1}(\lambda)\right) \mathbf{1}_{\left\{c>\zeta^{\prime-1}(\lambda)\right\}}-2\left(\zeta^{\prime-1}(2 \lambda)-c\right) \mathbf{1}_{\left\{c<\zeta^{\prime-1}(2 \lambda)\right\}}\right] \mathrm{d} \mu(c),
$$

which is a continuous function in $\lambda$. Also, $\xi\left(h_{\underline{\theta}}\right) \leq 0$ and $\xi\left(h_{\bar{\theta} / 2}\right) \geq 0$. Hence, there exists a $\lambda^{*} \in[\underline{\theta}, \bar{\theta} / 2]$ such that $\xi\left(h_{\lambda^{*}}\right)=0$. Note that if $\bar{\theta}=\infty$ then $\lim _{\lambda \uparrow \infty} \xi\left(h_{\lambda}\right)=\mathrm{E}[C]>0$. Thus, the $\lambda^{*}$ that solves $\xi\left(h_{\lambda}\right)=0$ is finite.

Using the observations of the above propositions with Lemma 2, we have the following result.

Theorem 2. Let $U^{*}$ be the optimal solution to (18). Then, $\lim _{n \rightarrow \infty} \rho_{n} \geq U^{*}$ with probability 1 .

Proof. Suppose that $\bar{\theta} \leq 2 \underline{\theta}$. Then, by Proposition $1, h^{*}$ solving (18) is $h^{*}(c)=c$. In this case, $U^{*}=\mathrm{E}[\zeta(C)]$ can be achieved via the flow profile $\left\{f_{v w}=C_{v w}\right\}_{v, w}$, which is shown to be feasible in Theorem 1 .

Now, suppose that $\bar{\theta}>2 \underline{\theta}$. We saw in Proposition 2 that there exists a

$$
\lambda^{*} \in\left[\underline{\theta}, \frac{1}{2} \bar{\theta}\right] \cap[0, \infty)
$$

with $\xi\left(h_{\lambda^{*}}\right)=0$, and

$$
h^{*}(c)=h_{\lambda^{*}}(c)=\operatorname{SAT}\left(c, \zeta^{\prime-1}\left(2 \lambda^{*}\right), \zeta^{\prime-1}\left(\lambda^{*}\right)\right) .
$$

Suppose that $\lambda^{*} \in(\underline{\theta}, \bar{\theta} / 2)$. Then, the optimising function $h^{*}(c)$ is bounded below by $\zeta^{\prime-1}\left(2 \lambda^{*}\right)>0$ and is bounded above by $\zeta^{\prime-1}\left(\lambda^{*}\right)<\infty$. Then, by Lemma 2 , the corresponding $U^{*}$ is achievable.

If $\lambda^{*}=\underline{\theta}$ then $\zeta^{\prime-1}\left(\lambda^{*}\right)=\infty$. Then, using (30), we have

$$
\xi\left(h_{\lambda^{*}}\right)=0 \quad \Longrightarrow \quad \operatorname{Pr}\left\{C<\zeta^{\prime-1}\left(2 \lambda^{*}\right)\right\}=0 .
$$

In this case, $h^{*}(c)=c$ over a set with probability 1 . Similarly, if $\lambda^{*}=\bar{\theta} / 2$ then $\zeta^{\prime-1}\left(2 \lambda^{*}\right)=0$, and, therefore, by (30), we have

$$
\xi\left(h_{\lambda^{*}}\right)=0 \quad \Longrightarrow \quad \operatorname{Pr}\left\{C>\zeta^{\prime-1}\left(\lambda^{*}\right)\right\}=0 .
$$

In this case also, $h^{*}(c)=c$ over a set with probability 1 . Hence, in the above two cases, $U^{*}$ is achievable via the flow profile $\left\{f_{v w}=h^{*}\left(C_{v w}\right)\right\}_{v, w}$, which is feasible with probability 1 . 


\section{Converse}

In this section we prove the converse of Theorem 2.

Theorem 3. Let $U^{*}$ be the optimal solution to (18). Then, $\lim \sup _{n \rightarrow \infty} \rho_{n} \leq U^{*}$ with probability 1 .

Proof. First, we proceed as in [1] to get a necessary condition for any flow (see (33), below). Consider an arbitrary capacity realisation $\left\{C_{v w}\right\}_{v, w}$. For any pair $\{v, w\}$, we have

$$
f_{v w}=\sum_{\{e: e \text { incident on } v\}} \varphi_{v w}(e),
$$

and, therefore,

$$
\sum_{e} \varphi_{v w}(e) \geq f_{v w}
$$

For pairs $\{v, w\}$ such that $f_{v w}>C_{v w}$, since at least $f_{v w}-C_{v w}$ flow has to be carried by a path of length two or more, we have the stronger condition

$$
\sum_{e} \varphi_{v w}(e) \geq C_{v w}+2\left(f_{v w}-C_{v w}\right)
$$

Combining (31) and (32) we have

$$
\sum_{e} \varphi_{v w}(e) \geq \min \left\{f_{v w}, C_{v w}\right\}+2\left(f_{v w}-C_{v w}\right)^{+} .
$$

Summing over all $\{v, w\}$ pairs and using (2), we obtain

$$
\sum_{e} C(e) \geq \sum_{\{v, w\}}\left(\min \left\{f_{v w}, C_{v w}\right\}+2\left(f_{v w}-C_{v w}\right)^{+}\right) .
$$

Division by $a_{n}$ and rearrangement yields

$$
\frac{1}{a_{n}} \sum_{\{v, w\}}\left(\left(C_{v w}-f_{v w}\right)^{+}-2\left(f_{v w}-C_{v w}\right)^{+}\right) \geq 0,
$$

a necessary condition for any flow $\left\{f_{v w}\right\}_{v, w}$ to be feasible. This was obtained by Aldous $e t$ $a l$. [1] in the context of uniform multicommodity flow with $f_{v w}=\phi$ for all $\{v, w\}$. But we see that (33) holds for any feasible flow.

It follows that $\rho_{n}$ is always less than or equal to the solution of the following optimisation problem:

$$
\max \frac{1}{a_{n}} \sum_{\{v, w\}} \zeta\left(f_{v w}\right)
$$

subject to

$$
\frac{1}{a_{n}} \sum_{\{v, w\}}\left(\left(C_{v w}-f_{v w}\right)^{+}-2\left(f_{v w}-C_{v w}\right)^{+}\right) \geq 0 .
$$


Let $\hat{U}$ be the optimal solution to this problem. Let $f=\left\{f_{v w}\right\}_{v, w}$, and define

$$
\begin{gathered}
\hat{\psi}(f)=\frac{1}{a_{n}} \sum_{\{v, w\}} \zeta\left(f_{v w}\right), \\
\hat{\xi}(f)=\frac{1}{a_{n}} \sum_{\{v, w\}}\left(\left(C_{v w}-f_{v w}\right)^{+}-2\left(f_{v w}-C_{v w}\right)^{+}\right) .
\end{gathered}
$$

For $\lambda>0$, independent of the realisation, define $\hat{w}(f, \lambda)=\hat{\psi}(f)+\lambda \hat{\xi}(f)$. We first optimise $\hat{w}(f, \lambda)$ for a fixed $\lambda>0$.

Using (34) and (35), we obtain

$$
\hat{w}(f, \lambda)=\frac{1}{a_{n}} \sum_{\{v, w\}}\left(\zeta\left(f_{v w}\right)+\lambda\left(C_{v w}-f_{v w}\right)^{+}-2 \lambda\left(f_{v w}-C_{v w}\right)^{+}\right) .
$$

The maximisation of $\hat{w}(f, \lambda)$ is separable in $\{v, w\}$, and, therefore, we optimise the summand for each $\{v, w\}$ by choosing an appropriate $f_{v w}$.

Comparing with the optimisation of $w(h, \lambda)$ in Section 4 , the optimising flow $f_{\lambda}$ is of the same form, i.e. $f_{\lambda, v w}=h_{\lambda}\left(C_{v w}\right)$.

If $\bar{\theta} \leq 2 \underline{\theta}$ then, for $\lambda$ such that $\bar{\theta} / 2 \leq \lambda \leq \underline{\theta}, f_{\lambda}$ is given by $f_{\lambda, v w}=C_{v w}$ for all $\{v, w\}$, as obtained in the proof of Proposition 1. In this case, $\hat{\xi}\left(f_{\lambda}\right)=0$ and

$$
\hat{\psi}\left(f_{\lambda}\right)=\frac{1}{a_{n}} \sum_{\{v, w\}} \zeta\left(C_{v w}\right)
$$

Thus,

$$
\limsup _{n \rightarrow \infty} \rho_{n} \leq \limsup _{n \rightarrow \infty} \frac{1}{a_{n}} \sum_{\{v, w\}} \zeta\left(C_{v w}\right) .
$$

The right-hand side is almost surely $\mathrm{E}[\zeta(C)]$, which is equal to $U^{*}$ in this case, and so $\lim \sup _{n \rightarrow \infty} \rho_{n} \leq U^{*}$ with probability 1 .

If $\bar{\theta}>2 \underline{\theta}$, choose $\lambda=\lambda^{*} \in[\underline{\theta}, \bar{\theta} / 2] \cap[0, \infty)$ so that $\xi$ as defined in (19) satisfies $\xi\left(h_{\lambda^{*}}\right)=0$ (note the distinction between $\xi$ and $\hat{\xi}$ ). As discussed in Proposition 2, such a $\lambda^{*}$ exists and is independent of the realisation with which we are now working. With this $\lambda^{*}, f_{\lambda^{*}, v w}=$ $h_{\lambda^{*}}\left(C_{v w}\right)=h^{*}\left(C_{v w}\right)$.

Now,

$$
\hat{w}\left(f_{\lambda^{*}}, \lambda^{*}\right) \geq \hat{w}\left(f, \lambda^{*}\right)
$$

which implies that

$$
\hat{\psi}\left(f_{\lambda^{*}}\right)+\lambda^{*} \hat{\xi}\left(f_{\lambda^{*}}\right) \geq \hat{\psi}(f)+\lambda^{*} \hat{\xi}(f)
$$

and, therefore,

$$
\hat{\psi}(f) \leq \hat{\psi}\left(f_{\lambda^{*}}\right)-\lambda^{*}\left(\hat{\xi}(f)-\hat{\xi}\left(f_{\lambda^{*}}\right)\right) .
$$

Hence, for all flow profiles $f$ that satisfy $\hat{\xi}(f) \geq 0$, we have

$$
\hat{\psi}(f) \leq \hat{\psi}\left(f_{\lambda^{*}}\right)+\lambda^{*} \hat{\xi}\left(f_{\lambda^{*}}\right)
$$

which implies that

$$
\rho_{n} \leq \hat{U} \leq \hat{\psi}\left(f_{\lambda^{*}}\right)+\lambda^{*} \hat{\xi}\left(f_{\lambda^{*}}\right)
$$


Note that

$$
\hat{\psi}\left(f_{\lambda^{*}}\right)=\frac{1}{a_{n}} \sum_{\{v, w\}} \zeta\left(h^{*}\left(C_{v w}\right)\right),
$$

which converges to $\mathrm{E}\left[\zeta\left(h^{*}(C)\right)\right]=U^{*}$ with probability 1 . Also,

$$
\hat{\xi}\left(f_{\lambda^{*}}\right)=\frac{1}{a_{n}} \sum_{\{v, w\}}\left(\left(C_{v w}-h^{*}\left(C_{v w}\right)\right)^{+}-2\left(h^{*}\left(C_{v w}\right)-C_{v w}\right)^{+}\right),
$$

which converges to

$$
\mathrm{E}\left[\left(C-h^{*}(C)\right)^{+}-2\left(h^{*}(C)-C\right)^{+}\right]=\xi\left(h^{*}\right)=0,
$$

with probability 1 . Thus, taking $\lim$ sup in (36), we have $\lim \sup _{n \rightarrow \infty} \rho_{n} \leq U^{*}$, and the proof is complete.

\section{Conclusion}

We studied the asymptotic behaviour of optimal flows on the complete graph. The optimal net utility converges with probability 1 to a value that depends on the distribution of $C$. Interestingly, the volume of each flow depends only on the capacity of the corresponding direct link via a simple function. More precisely, we have shown the following.

1. If the slope of the utility function $\zeta$ at the origin is less than twice the slope at infinity, i.e. $\bar{\theta} \leq 2 \underline{\theta}$, then $\lim _{n \rightarrow \infty} \rho_{n}=\mathrm{E}[\zeta(C)]$ with probability 1 , and it is optimal to route each flow entirely via the direct link.

2. If $\bar{\theta}>2 \underline{\theta}$ then $\lim _{n \rightarrow \infty} \rho_{n}=\mathrm{E}\left[\zeta\left(h^{*}(C)\right)\right]$ with probability 1 , where

$$
h^{*}(c)=\operatorname{SAT}\left(c, \zeta^{\prime-1}\left(2 \lambda^{*}\right), \zeta^{\prime-1}\left(\lambda^{*}\right)\right)
$$

and $\lambda^{*}$ solves

$$
\mathrm{E}\left[\left(C-h^{*}(C)\right)^{+}\right]-2 \mathrm{E}\left[\left(h^{*}(C)-C\right)^{+}\right]=0 .
$$

The flow for each pair $\{v, w\}$ is $h^{*}\left(C_{v w}\right)$ and is routed through only direct and two-hop routes. The resultant flow profile can be obtained through a simple distributed algorithm that requires information sharing only among links that share a vertex.

\section{References}

[1] Aldous, D. J., McDiarmid, C. And Scott, A. (2009). Uniform multicommodity flow through the complete graph with random edge-capacities. Operat. Res. Lett. 37, 299-302.

[2] Chayes, J. T. and Chayes, L. (1986). Bulk transport properties and exponent inequalities for random resistor and flow networks. Commun. Math. Phys. 105, 133-152.

[3] Georgiadis, L., Neely, M. J. and Tassiulas, L. (2006). Resource allocation and cross-layer control in wireless networks. Foundations Trends Networking 1, 1-144.

[4] Grimmett, G. and Kesten, H. (1984). First-passage percolation, network flows and electrical resistances. Z. Wahrscheinlichkeitsth. 66, 335-366.

[5] Grimmett, G. R. and Stirzaker, D. R. (1992). Probability and Random Processes, 2nd edn. Clarendon Press, Oxford.

[6] Kapoor, S. And VaIdya, P. M. (1996). Speeding up Karmarkar's algorithm for multicommodity flows. Math. Program. A 73, 111-127.

[7] Kelly, F. P., Maulloo, A. K. and Tan, D. K. H. (1998). Rate control for communication networks: shadow prices, proportional fairness and stability. J. Operat. Res. Soc. 49, 237-252. 
[8] Leighton, T. et al. (1995). Fast approximation algorithms for multicommodity flow problems. J. Comput. System Sci. 50, 228-243.

[9] Megiddo, N. (1974). Optimal flows in networks with multiple sources and sinks. Math. Program. 7, 97-107.

[10] Mo, J. and Walrand, J. (2000). Fair end-to-end window-based congestion control. IEEE/ACM Trans. Networking 8, 556-567.

[11] Zhang, Y. (2000). Critical behavior for maximal flows on the cubic lattice. J. Statist. Phys. 98, $799-811$. 